\title{
Innovación docente aplicada a las prácticas de laboratorio de la asignatura Ingeniería de Superficies.
}

\section{Teaching innovation applied to laboratory practices of Surface Engineering}

ANTONIO PEREjón PAZO

ORCID: https://orcid.org/0000-0002-5525-2227

Universidad de Sevilla

Departamento de Química Inorgánica

aperejon@us.es

Fecha de recepción:

Fecha de aceptación:

DOI: http://dx.doi.org/10.12795/9788447221912.107

Pp.: 2396-2418 


\section{Resumen:}

El Ciclo de Mejora se ha aplicado a las prácticas de la asignatura Ingeniería de Superficies correspondiente al primer cuatrimestre del Cuarto Curso del Grado en Ingeniería de Materiales y Quinto Curso del Doble Grado en Física e Ingeniería de Materiales y Doble Grado en Química e Ingeniería de Materiales. De las 15 horas de prácticas de las que se dispone en esta asignatura, el Ciclo de Mejora ha abarcado 9 horas, correspondientes a las sesiones de 3 horas de duración impartidas en los tres primeros días de prácticas. El modelo metodológico se ha diseñado con el objetivo de impulsar la creatividad de los estudiantes. Así, las prácticas se han enfocado de forma que serán los estudiantes quienes diseñen los experimentos necesarios para llegar a la solución o soluciones de un determinado problema.

Palabras clave: Ingeniería de Superficies, Grado en Ingeniería de Materiales, Doble Grado en Física e Ingeniería de Materiales, Doble Grado en Química e Ingeniería de Materiales, Docencia Universitaria, Experimentación Docente Universitaria.

\section{Abstract:}

A Teaching Improvement Cycle has been applied to laboratory practices of Surface Engineering, corresponding to the first term of the Fourth Course of the Degree in Materials Engineering and the Fifth Course of the Double Degree in Physics and Materials Engineering and the Double Degree in Chemistry and Materials Engineering. The Teaching Improvement Cycle has covered nine of the fifteen hours of laboratory practices available in this subject, which correspond to the 3-hour sessions taught in the three first days of practices. The methodological model has been designed with the objective of boosting creativity of the students. Thus, the laboratory practices have been focused in such a way that the students are responsible of designing the experiments needed to reach the solution or solutions of a certain problem.

Keywords: Surface Engineering, Degree in Materials Engineering, Double Degree in Physics and Materials Engineering, Double Degree in Chemistry and Materials Engineering, University Teaching, University Teaching Experimentation. 


\section{Contexto}

El Ciclo de Mejora tiene en cuenta que los estudiantes (16) se encuentran en cuarto y quinto curso, por lo que tienen experiencia previa en laboratorios de química y se les presupone cierta formación en el manejo de algunas técnicas instrumentales. Sin embargo, no han tenido que enfrentarse al diseño de una práctica de laboratorio para resolver un determinado problema.

Los conocimientos que pueden adquirir serán útiles cuando tengan que enfrentarse al mundo laboral, en el que deberán ser creativos y reflexivos para solucionar los problemas. Además, los estudiantes tendrán que sintetizar y analizar los resultados obtenidos, y deberán estudiar si éstos tienen sentido.

Se ha fomentado en las prácticas que los estudiantes tomen una actitud crítica a la hora de decidir qué pasos deben seguir para resolver el problema dado. Por tanto, uno de los principales objetivos del modelo metodológico es que los estudiantes razonen por sí mismos (Bain, 2007).

\section{Diseño previo del CIMA}

\section{Mapa de contenidos y problemas}

En la Figura 1 se presenta el mapa de contenidos asociado a las prácticas de laboratorio que se sometieron al Ciclo de Mejora. Estos contenidos se trabajaron en dos prácticas de laboratorio durante las 9 horas de aplicación del Ciclo de Mejora, en las que los estudiantes tuvieron que diseñar protocolos de trabajo para solucionar dos problemas, que se plantearon en los siguientes términos:

1. Práctica 1. Determina el tamaño de la molécula de trioleína (derivado del ácido oleico) a partir del 
material de laboratorio que se ha proporcionado: micropipeta, cubeta y agua. Diseña el experimento que permita estimar dicho tamaño.

2. Práctica 2. Imagina que trabajas en el Departamento de Calidad de una empresa de fabricación de galletas. Os informan de que las galletas que fabricáis en la empresa se rompen siempre en el camino hacia el punto de venta. Se os ocurre que podríais disminuir la fragilidad de las galletas recubriéndolas con chocolate. Diseña un procedimiento aplicable a escala de laboratorio para determinar si un recubrimiento de chocolate disminuye la fragilidad de las galletas. ; Qué parámetros se pueden modificar?

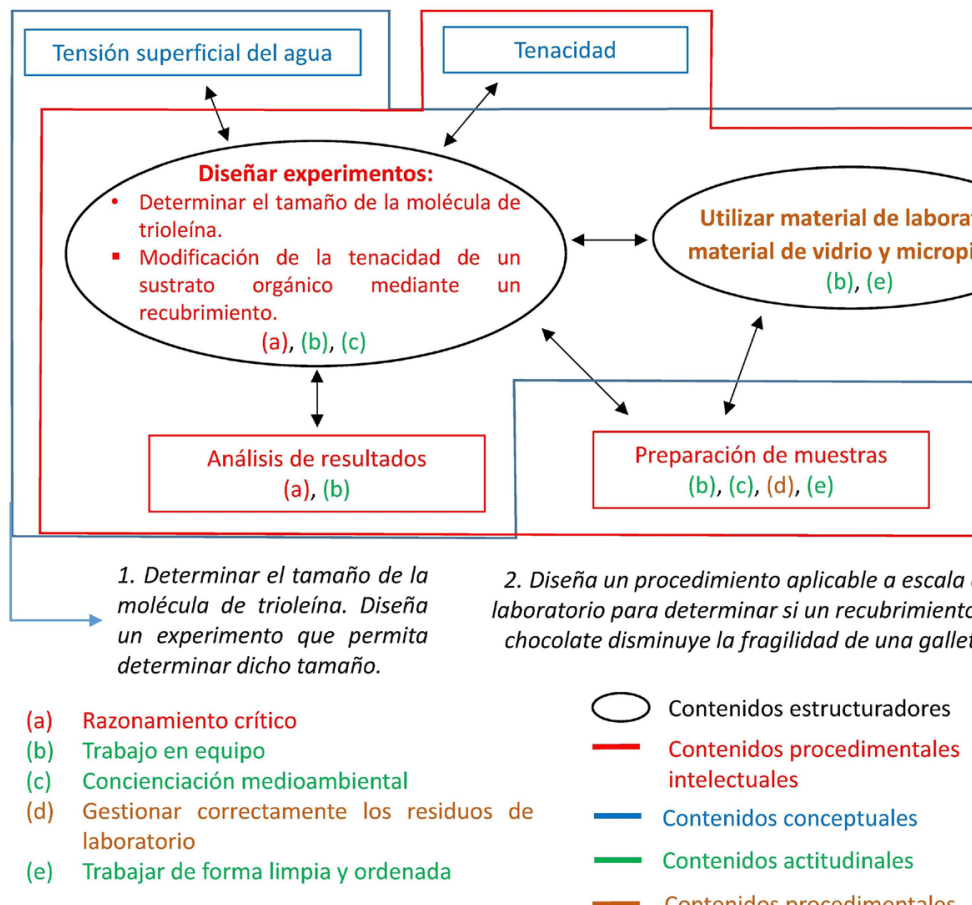

Figura 1. Mapa de contenidos y problemas.

Como se puede observar en el mapa de contenidos y problemas, los contenidos estructuradores están relacionados con el diseño de experimentos y la utilización de material de laboratorio. Estos dos contenidos son el 
eje central de las prácticas que se sometieron al Ciclo de Mejora. El diseño de experimentos es un procedimiento intelectual fundamental, ya que para que los estudiantes sean capaces de diseñar estos experimentos deben aplicar contenidos conceptuales (tensión superficial del agua, tenacidad) y también procedimentales, teniendo en cuenta que deben estar familiarizados con la forma de trabajar en un laboratorio. Una vez diseñadas las prácticas, los estudiantes las pondrán en práctica, para lo que tendrán que preparar muestras y analizar críticamente los resultados obtenidos (fundamentalmente procedimentales intelectuales).

\section{Modelo metodológico posible}

El modelo metodológico diseñado se presenta en la Figura 2 y consta de 5 fases. En primer lugar, se proporciona a los estudiantes, por parejas, el material necesario para la realización de la práctica, se les plantea el problema $(P R)$ y se les indica que tienen que diseñar un experimento para resolverlo.

A continuación, los estudiantes disponen de tiempo para estudiar diferentes posibilidades para diseñar el experimento (IA). Seguidamente, se entregan textos que sirven de orientación para llegar a la solución. El profesor supervisa las discusiones de los estudiantes para el diseño de los experimentos e interviene para guiarlos hacia el diseño correcto para la resolución del problema (AC). Se realiza la práctica siguiendo los pasos propuestos en el diseño (IA) y, finalmente, se hace una puesta en común en la que cada pareja presenta su solución al problema propuesto (PC). El modelo metodológico se aplica a las dos prácticas contenidas en el Ciclo de Mejora. 
PR: Problema

IA: Ideas de los alumnos

AC: Actividad de contraste

PC: Puesta en común

\section{IA}

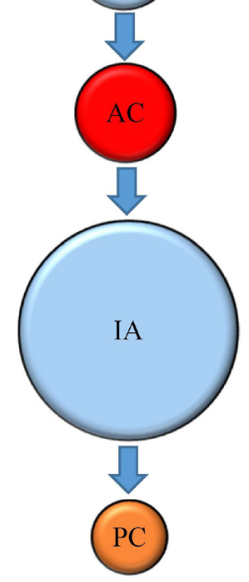

Figura 2. Modelo metodológico posible.

\section{Secuencia de actividades}

En las Tablas 1-3 se muestra, en orden cronológico, la secuencia de actividades planeada para cada sesión de prácticas de tres horas.

Jornadas de Formación e Innovación Docente del Profesorado I № 2 (2019) 
Tabla 1. Secuencia de actividades correspondiente a la primera sesión de prácticas.

\begin{tabular}{|c|c|c|c|}
\hline \multicolumn{4}{|c|}{ SESIÓN 1. Práctica 1. ¿Cuál es el tamaño de la molécula de trioleína? } \\
\hline Actividad & Descripción & Tiempo & Fase \\
\hline $\begin{array}{l}\text { № 1: Presentación } \\
\text { de la Práctica } 1\end{array}$ & $\begin{array}{l}\text { Se presentan brevemente las dos prácticas } \\
\text { que se van a realizar en las tres primeras } \\
\text { sesiones de prácticas. A continuación, se inicia } \\
\text { la Práctica 1. Se entrega a los estudiantes un } \\
\text { guion donde se indica el título de la práctica } \\
\text { ("El tamaño de las moléculas”), el material que } \\
\text { se va a emplear y el problema que hay que } \\
\text { resolver en esta práctica: Determinar el tamaño } \\
\text { de la molécula de trioleína (derivado del ácido } \\
\text { oleico) a partir del material de laboratorio } \\
\text { que se ha proporcionado: micropipeta, cubeta } \\
\text { y agua. Diseña un experimento que permita } \\
\text { estimar dicho tamaño. }\end{array}$ & $15^{5}$ & PR \\
\hline \multicolumn{4}{|c|}{ Recursos: Intervención del profesor y guion de prácticas en papel. } \\
\hline $\begin{array}{l}\text { № 2: } \\
\text { Ideas de los } \\
\text { estudiantes }\end{array}$ & $\begin{array}{l}\text { Los estudiantes empiezan a debatir, por } \\
\text { parejas, el posible diseño experimental } \\
\text { adecuado para solucionar el problema } \\
\text { propuesto. El profesor escucha las ideas de } \\
\text { los estudiantes y sólo interviene si lo estima } \\
\text { oportuno para orientarlos. }\end{array}$ & $30^{-}$ & IA \\
\hline \multicolumn{4}{|c|}{ Recursos: Guion de prácticas, intervenciones puntuales del profesor. } \\
\hline $\begin{array}{l}\text { № 3: } \\
\text { Texto } \\
\text { complementario y } \\
\text { diseño }\end{array}$ & $\begin{array}{l}\text { Tras escuchar las ideas de los estudiantes, el } \\
\text { profesor entrega copias de una carta en la que } \\
\text { Benjamin Franklin describe los experimentos } \\
\text { que llevó a cabo añadiendo aceite al agua } \\
\text { (Franklin, Brownrigg y Farish 1774). Los } \\
\text { estudiantes leen la carta, que sirve como } \\
\text { complemento al problema planteado. Los } \\
\text { estudiantes muestran al profesor el protocolo } \\
\text { diseñado para la práctica, quien los guia para } \\
\text { que lleguen a un diseño final correcto. }\end{array}$ & $30^{-}$ & $A C$ \\
\hline \multicolumn{4}{|c|}{$\begin{array}{l}\text { Recursos: Carta de Benjamin Franklin, ideas de los estudiantes e intervenciones del } \\
\text { profesor. }\end{array}$} \\
\hline $\begin{array}{l}\text { № 4: } \\
\text { Realización de la } \\
\text { práctica }\end{array}$ & $\begin{array}{l}\text { Los estudiantes realizan la práctica siguiendo } \\
\text { los pasos descritos en el diseño. Repiten } \\
\text { la práctica varias veces para obtener una } \\
\text { estadística de los resultados obtenidos. } \\
\text { Además, realizan los cálculos necesarios para } \\
\text { obtener el tamaño de la molécula de trioleína. }\end{array}$ & $75^{\prime}$ & IA \\
\hline
\end{tabular}


Recursos: Material de laboratorio proporcionado a los estudiantes. \begin{tabular}{l|l} 
No 5: & Los estudiantes muestran los resultados
\end{tabular} Puesta en común obtenidos y se discuten. Se enfatiza en que un diseño sencillo permite una estimación muy aproximada del tamaño de la molécula de trioleína.

Recursos: Pizarra y resultados de los estudiantes.

Tabla 2. Secuencia de actividades correspondiente a la segunda sesión de prácticas.

\begin{tabular}{|c|c|c|c|}
\hline \multicolumn{4}{|c|}{$\begin{array}{c}\text { SESIÓN 2. Práctica 2. Diseñar un procedimiento aplicable a escala de laboratorio } \\
\text { para determinar si un recubrimiento de chocolate disminuye la fragilidad de } \\
\text { unas galletas. Primera Parte. }\end{array}$} \\
\hline Actividad & Descripción & Tiempo & Fase \\
\hline $\begin{array}{l}\text { № 6: } \\
\text { Presentación de la } \\
\text { práctica } 2\end{array}$ & $\begin{array}{l}\text { Se entrega a los estudiantes un guion } \\
\text { donde se indica el título de la segunda } \\
\text { práctica ("Estudio de los efectos de la } \\
\text { deposición de láminas delgadas orgánicas } \\
\text { sobre sustratos porosos en la energía de } \\
\text { ruptura del material final”), el material que } \\
\text { se va a emplear y el problema que hay que } \\
\text { resolver: Diseña un procedimiento aplicable } \\
\text { a escala de laboratorio para determinar si } \\
\text { un recubrimiento de chocolate disminuye la } \\
\text { fragilidad de las galletas. }\end{array}$ & $15^{\prime}$ & PR \\
\hline \multicolumn{4}{|c|}{ Recursos: Intervención del profesor y guion de prácticas en papel. } \\
\hline $\begin{array}{l}\text { № 7: } \\
\text { Ideas de los } \\
\text { estudiantes }\end{array}$ & $\begin{array}{l}\text { Los estudiantes debaten, por parejas, el } \\
\text { posible diseño experimental adecuado } \\
\text { para solucionar el problema propuesto. } \\
\text { El profesor escucha las ideas de los } \\
\text { estudiantes y sólo interviene si lo estima } \\
\text { oportuno para orientarlos. }\end{array}$ & $30^{-}$ & IA \\
\hline
\end{tabular}

Jornadas de Formación e Innovación Docente del Profesorado | № 2 (2019) Esta obra se distribuye con la licencia Creative Commons Reconocimiento-NoComercial-SinObraDerivada Internacional (CC BY-NC-ND 4.0.) 


\begin{tabular}{|c|c|c|c|}
\hline $\begin{array}{l}\text { № 8: } \\
\text { Péndulo Charpy y } \\
\text { diseño }\end{array}$ & $\begin{array}{l}\text { Se entrega un artículo en el que se describe } \\
\text { la historia del péndulo Charpy, ampliamente } \\
\text { empleado en estudios de tenacidad (o } \\
\text { fragilidad) de materiales, que sirve como } \\
\text { complemento al problema planteado } \\
\text { (Bayraktar, Kaplan, Schmidt, Paqueton y } \\
\text { Grumbach, 2008). Los estudiantes leen el } \\
\text { artículo y muestran al profesor el protocolo } \\
\text { diseñado para la práctica, quien los guía } \\
\text { para que lleguen a un diseño final correcto. } \\
\text { Se deben tener en cuenta parámetros } \\
\text { como la concentración o concentraciones } \\
\text { de chocolate con las que se van a realizar } \\
\text { los estudios, el tipo de disolvente (agua o } \\
\text { leche) y el procedimiento para recubrir las } \\
\text { galletas. }\end{array}$ & $60^{\circ}$ & $A C$ \\
\hline \multicolumn{4}{|c|}{$\begin{array}{l}\text { Recursos: Artículo sobre el péndulo Charpy, ideas de los estudiantes e intervenciones } \\
\text { del profesor. }\end{array}$} \\
\hline $\begin{array}{l}\text { № 9: Preparación de } \\
\text { muestras }\end{array}$ & $\begin{array}{l}\text { Se preparan las disoluciones de chocolate y } \\
\text { se recubren las galletas, que se dejan secar } \\
\text { hasta la siguiente sesión. }\end{array}$ & $75^{\prime}$ & IA \\
\hline
\end{tabular}

Tabla 3. Secuencia de actividades correspondiente a la tercera sesión de prácticas.

\begin{tabular}{|c|c|c|c|}
\hline \multicolumn{4}{|c|}{$\begin{array}{l}\text { SESIÓN 3. Práctica 2. Diseñar un procedimiento aplicable a escala de laboratorio } \\
\text { para determinar si un recubrimiento de chocolate disminuye la fragilidad de } \\
\text { unas galletas. Segunda Parte. }\end{array}$} \\
\hline Actividad & Descripción & Tiempo & Fase \\
\hline $\begin{array}{l}\text { № 10: } \\
\text { Montaje experimental }\end{array}$ & $\begin{array}{l}\text { Los estudiantes realizan un montaje } \\
\text { experimental de un péndulo charpy } \\
\text { "casero", que sirve para llevar a cabo } \\
\text { los ensayos de tenacidad de las galletas } \\
\text { recubiertas de chocolate. Además, hacen } \\
\text { algunas pruebas previas para asegurar el } \\
\text { correcto funcionamiento del péndulo. }\end{array}$ & $60^{\circ}$ & IA \\
\hline
\end{tabular}

Jornadas de Formación e Innovación Docente del Profesorado | № 2 (2019) Esta obra se distribuye con la licencia Creative Commons Reconocimiento-NoComercial-SinObraDerivada Internacional (CC BY-NC-ND 4.0.) 


\begin{tabular}{|l|l|l|l|}
\hline № 11: & $\begin{array}{l}\text { Los estudiantes hacen los ensayos de } \\
\text { Ensayos de tenacidad de las galletas recubiertas, } \\
\text { repitiendo los experimentos varias } \\
\text { veces para hacer una estadística de los } \\
\text { resultados. Además, realizan los cálculos } \\
\text { necesarios para obtener la tenacidad de } \\
\text { las galletas recubiertas de chocolate en } \\
\text { relación con las galletas sin recubrir. }\end{array}$ & IA \\
\hline Recursos: Muestras y material de laboratorio. & PC \\
\hline № 12: & $\begin{array}{l}\text { Los estudiantes muestran los resultados } \\
\text { que han obtenido y se discuten. Se enfatiza } \\
\text { en que los resultados obtenidos son una } \\
\text { aproximación, pero que son comparables } \\
\text { entre sí para diferentes concentraciones y } \\
\text { recubrimientos. }\end{array}$ & $30^{\prime}$ & \\
\hline
\end{tabular}

\section{Cuestionario inicial-final}

Teniendo en cuenta los conceptos teóricos principales que se van a trabajar en las prácticas de laboratorio, descritos en el mapa de contenidos y problemas (Figura 1) y desarrollados en la secuencia de actividades, se elaboró un cuestionario que se entregó a los estudiantes antes y después de la realización de las prácticas, con el objetivo de conocer las ideas iniciales de los estudiantes y su evolución tras la impartición de las prácticas (Porlán, 2017). Las preguntas que se incluyeron en el cuestionario fueron las siguientes:

1. Estás sentado en un parque delante de un estanque en un día de primavera. Contemplas el vuelo de un mosquito que acaba posándose en el agua del estanque, ¿por qué el mosquito no se hunde en el agua?

2. Observas unas gotas de rocío sobre unas hojas cercanas al estanque, ¿podrías decir por qué son aproximadamente esféricas?

3. Divisas a lo lejos un camión cisterna que está vertiendo aceite al estanque, ¿qué ocurrirá con el vertido? ¿Por qué? 
4. En el parque, unos niños están jugando en un columpio, ¿por qué se utilizan cadenas de acero para sostener el columpio?

5. ¿Qué otros materiales se podrían usar para sostener el columpio y cuáles no? ¿Por qué?

Las preguntas 1-3 pretenden evaluar las ideas iniciales y finales de los estudiantes con respecto al fenómeno de la tensión superficial, con el que se trabaja en la práctica relacionada con la determinación del tamaño de la molécula de trioleína. Las preguntas 4-5 están enfocadas en el concepto de tenacidad, aplicado en la práctica de determinación de la fragilidad de una galleta recubierta de chocolate. En la Tabla 4 se relacionan las cuestiones planteadas con los conceptos y los problemas asociados.

Tabla 4. Relación Cuestionario-Problemas-Mapa de contenidos

\begin{tabular}{|c|c|c|}
\hline Cuestión & Concepto relacionado & Problema \\
\hline 1 & Tensión superficial del agua & $\begin{array}{c}\text { Determinación del tamaño de la } \\
\text { molécula de trioleína }\end{array}$ \\
\hline 2 & Tensión superficial del agua & $\begin{array}{c}\text { Determinación del tamaño de la } \\
\text { molécula de trioleína }\end{array}$ \\
\hline 3 & Tensión superficial del agu. & $\begin{array}{c}\text { Determinación del tamaño de la } \\
\text { molécula de trioleín. }\end{array}$ \\
\hline 4 & Tenacidad & $\begin{array}{c}\text { Determinación de la fragilidad de } \\
\text { una galleta recubierta de chocolate }\end{array}$ \\
\hline 5 & Tenacidad & $\begin{array}{c}\text { Determinación de la fragilidad de } \\
\text { una galleta recubierta de chocolate }\end{array}$ \\
\hline
\end{tabular}

Jornadas de Formación e Innovación Docente del Profesorado | № 2 (2019) Esta obra se distribuye con la licencia Creative Commons Reconocimiento-NoComercial-SinObraDerivada Internacional (CC BY-NC-ND 4.0.) 


\section{Aplicación del CIMA}

\section{Relato resumido de las sesiones}

- Primera sesión

Los primeros minutos de la primera sesión se dedicaron a presentar brevemente las dos prácticas que se iban a realizar en las tres primeras sesiones y a recordar las normas de seguridad en el laboratorio. Así, se indicó a los estudiantes que se les expondrían dos problemas que tendrían que solucionar mediante el diseño de dos prácticas, y que la única información de la que dispondrían sería un pequeño guion con el material de laboratorio que iban a usar en cada práctica y la descripción del problema a resolver. Debido a que estas prácticas se enfocaron de manera que sean los estudiantes quienes diseñen los procedimientos para llegar a la solución de los problemas, se propuso que sólo pudiesen hacer uso de los teléfonos móviles cuando el profesor les diese permiso, mientras tanto debían permanecer guardados. A los estudiantes les pareció bien la propuesta, por lo que se aceptó y se pasó a la realización de la primera práctica.

Se entregó el guion y se indicó a los estudiantes que debían tener en cuenta los conceptos teóricos relacionados con el problema a resolver y además describir paso a paso el procedimiento experimental que permitiese llegar a la solución. Los estudiantes empezaron a debatir por parejas y se les dijo que cuando llegasen a alguna conclusión acerca de los conceptos teóricos a aplicar y el diseño del procedimiento experimental, debían explicárselo al profesor. De esta forma, el profesor podía escuchar las primeras ideas de todos los estudiantes.

Tras unos treinta minutos de debate, se pudo comprobar que muchos estudiantes habían avanzado bastante tanto en los conceptos teóricos como en posibles 
procedimientos experimentales a aplicar, de forma que se crearon debates interesantes no sólo entre los estudiantes sino también entre los estudiantes y el profesor. Se realizó entonces la actividad de contraste, consistente en la entrega de una carta de Benjamin Franklin en la que él mismo describe sus observaciones al añadir aceite al agua (Franklin, Brownrigg y Farish 1774), y los estudiantes dedicaron unos minutos a leerla con detenimiento para contraponer lo que se dice en la carta con sus primeras ideas. A partir de la lectura de la carta, casi todos los estudiantes fueron capaces de enfocar de nuevo los diseños experimentales y con la orientación del profesor fueron capaces de llegar a una descripción correcta del protocolo de trabajo que permite llegar a la solución del problema.

A continuación, los estudiantes se dedicaron a realizar la parte experimental de la práctica. Debido a que era dificil llevar a cabo los experimentos con exactitud con los medios disponibles, se encontró inicialmente una gran disparidad de resultados, por lo que se les indicó a los estudiantes que debían repetir la práctica todas las veces que pudiesen en el tiempo del que disponían, de forma que se pudiese obtener una estadística de los resultados. A medida que los estudiantes repitieron la práctica y adquirieron destreza en el procedimiento experimental, los resultados fueron cada vez menos dispares y relativamente cercanos a los valores esperados.

Finalmente, los últimos minutos de la sesión se dedicaron a escribir en la pizarra los resultados obtenidos por cada pareja. Debido a que se repitieron muchas veces los experimentos, la actividad de puesta en común tuvo que reducirse con respecto al tiempo planeado. Se comprobó que en casi todos los casos se había llegado a resultados similares, aunque estaban un poco alejados del resultado esperado. Esta diferencia se explicó teniendo en cuenta que el diseño experimental daba lugar a ciertos errores en cada paso, que colectivamente producían un error experimental considerable. 
La experiencia fue muy buena con esta práctica, ya que los estudiantes se implicaron desde el principio en realizarla correctamente y además se sorprendieron de que se pudiese llegar a resultados aceptables a partir de un experimento sencillo.

\section{- Segunda sesión}

La segunda práctica, por ser más extensa en cuanto al procedimiento experimental a llevar a cabo, se realizó en la segunda y tercera sesiones. Se inició la segunda sesión con la entrega del guion correspondiente a la segunda práctica. Se explicó que, al igual que en la primera práctica, los estudiantes debían tener en cuenta ciertos conceptos teóricos necesarios para llegar a un diseño correcto. A continuación, los estudiantes debatieron por parejas sobre el contenido teórico a aplicar y el procedimiento experimental que debían llevar a cabo. Tras escuchar las ideas de los estudiantes, se comprobó que para esta práctica tenían más claros los conceptos teóricos que para la práctica anterior, pero avanzaban más lentamente en el diseño del procedimiento experimental, que en este caso era más largo.

Se entregó a continuación un artículo descriptivo de la historia del péndulo Charpy (Bayraktar, Kaplan, Schmidt, Paqueton y Grumbach, 2008), con el objetivo de que los estudiantes tuviesen clara la importancia de este tipo de ensayos en el estudio de la tenacidad de los materiales. Durante aproximadamente una hora, los estudiantes leyeron el artículo y continuaron con el diseño del protocolo a seguir para llevar a cabo el experimento. Se insistió a los estudiantes que la idea era que fuesen capaces ellos mismos de diseñar la receta de la práctica de forma que un estudiante que no la conociese fuese capaz de reproducirla. Finalmente, con las orientaciones del profesor, los estudiantes llegaron a un diseño adecuado, y se consensuaron las concentraciones y tipo de disolvente a utilizar. 
La última parte de la sesión se dedicó a la preparación de disoluciones con diferente concentración de chocolate para comprobar su efecto en la resistencia al impacto de las galletas recubiertas. Se recubrieron las galletas con hasta cuatro capas de chocolate para cada concentración, y se dejaron secar hasta la siguiente sesión.

- Tercera sesión

Esta sesión fue fundamentalmente experimental. Así, en la primera parte de la sesión los estudiantes montaron tres péndulos Charpy "caseros" y estudiaron la forma más adecuada de realizar las medidas de tenacidad realizando pruebas con galletas sin recubrir. Quisiera destacar la buena implicación de los estudiantes en la realización correcta de las medidas.

Seguidamente, se llevaron a cabo los ensayos de tenacidad con los péndulos optimizados sobre las galletas recubiertas con chocolate a diferentes concentraciones y con diferentes capas. Se hicieron varios ensayos para cada concentración con el objetivo de hacer una pequeña estadística de los resultados y descartar aquellos que se desviaban demasiado de la media. Tras obtener los datos experimentales, se pasó al cálculo de la tenacidad (o resiliencia) de las galletas, en función de la concentración de chocolate de las disoluciones con las que se hicieron los recubrimientos y teniendo en cuenta el espesor de chocolate depositado. En general, los estudiantes fueron capaces de realizar los cálculos adecuadamente, y las dudas que surgieron se resolvieron sin dificultades.

Finalmente, se hizo un cuadrante en la pizarra en la que cada pareja de estudiantes mostró los resultados obtenidos en función de la concentración de chocolate y del número de capas. Se estableció un pequeño debate en el que se preguntó a los estudiantes si los resultados tenían sentido a pesar de haber trabajado con un péndulo Charpy "casero". Se comprobó que la resistencia al choque de las galletas aumentaba con la concentración de chocolate y con el número de capas. 
Sin embargo, se observó que para un grupo de estudiantes los resultados no concordaban teniendo en cuenta la concentración y número de capas de chocolate. Revisando los recubrimientos, se comprobó que se había formado una capa de polímero en las galletas debido a que habían calentado la disolución a temperatura demasiado alta y el almidón de maíz que lleva el chocolate comercial había polimerizado.

Se insistió a los estudiantes que cuando un resultado experimental se desvía del resto es importante tratar de buscarle una explicación antes de descartarlo. Terminamos la sesión resolviendo algunas pequeñas dudas finales.

\section{Evaluación del aprendizaje de los estudiantes. Escaleras de Aprendizaje.}

Los estudiantes respondieron al cuestionario indicado en el epígrafe anterior antes y después de la realización de las prácticas de laboratorio. Teniendo en cuenta las respuestas se elaboró una escalera de aprendizaje para cada pregunta, en la que se incluyen los modelos de ideas iniciales y finales y el porcentaje de estudiantes que respondieron en base a cada idea (Porlán, 2017). En las escaleras, los porcentajes en negro hacen referencia a la cantidad de estudiantes que respondieron una misma idea inicial, y los porcentajes en rojo a la cantidad de estudiantes que respondieron en base a una misma idea final. Las escaleras contienen cuatro niveles o escalones (A-D) o tres niveles $(A-C)$ en función de las ideas aportadas. Junto a cada escalón, se indica el obstáculo de aprendizaje que los estudiantes deben superar para alcanzar el siguiente nivel.

En la Figura 3 se muestra, a modo de ejemplo, la escalera de aprendizaje elaborada a partir de la cuestión 3: "Divisas a lo lejos un camión cisterna que está vertiendo aceite al estanque, ¿qué ocurrirá con el vertido? ¿Por qué?". Las ideas de los estudiantes se distribuyeron en 
tres niveles. La mayoría de los estudiantes consideró inicialmente que el aceite queda por encima del agua, pero sin especificar la interacción agua-aceite que hace que se forme una capa de aceite sobre el agua. Tras la realización de las prácticas, un $82 \%$ de estudiantes fue capaz de responder en base a dicha interacción.

La Tabla 5 incluye los avances individuales de los estudiantes. Se muestra el número de niveles que superó cada estudiante para cada cuestión entre los cuestionarios inicial y final. Se aprecia que prácticamente todos avanzaron en alguna de las cuestiones, y en algunos casos los cambios son muy significativos. Los estudiantes que presentan menos avances son aquellos que habían llegado a los niveles más altos en las respuestas aportadas para los cuestionarios iniciales. Se comprueba que los avances más importantes se produjeron para las cuestiones 3 y 4.

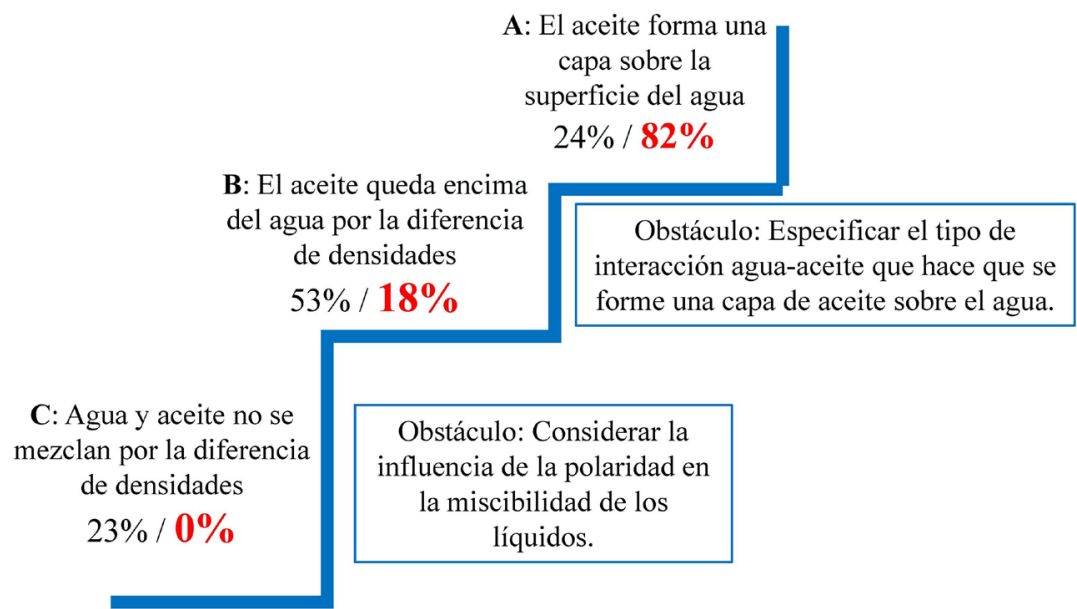

Figura 3. Escalera de aprendizaje elaborada a partir de las respuestas de los estudiantes a la cuestión 3. 
Tabla 5. Avances individuales de los estudiantes a partir de las escaleras de aprendizaje.

\begin{tabular}{|c|c|c|c|c|c|}
\hline Estudiante & Cuestión 1 & Cuestión 2 & Cuestión 3 & Cuestión 4 & Cuestión 5 \\
\hline 1 & 0 & +1 & 0 & 0 & 0 \\
\hline 2 & 0 & 0 & +1 & +1 & 0 \\
\hline 3 & 0 & +1 & +3 & +2 & +1 \\
\hline 4 & +1 & +1 & +1 & 0 & +1 \\
\hline 5 & +2 & 0 & +1 & +3 & +2 \\
\hline 6 & 0 & 0 & +3 & 0 & 0 \\
\hline 7 & 0 & +2 & +1 & +2 & +2 \\
\hline 8 & 0 & +1 & 0 & +2 & 0 \\
\hline 9 & 0 & 0 & +1 & +1 & +1 \\
\hline 10 & 0 & 0 & 0 & 0 & +1 \\
\hline 11 & +2 & 0 & 0 & 0 & 0 \\
\hline 12 & 0 & +1 & +3 & +1 & +1 \\
\hline 13 & 0 & +2 & +1 & 0 & 0 \\
\hline 14 & 0 & 0 & 0 & 0 & 0 \\
\hline 15 & +1 & +1 & 0 & 0 & +2 \\
\hline 16 & +2 & +1 & +1 & +2 & +1 \\
\hline
\end{tabular}

Los resultados obtenidos indican la necesidad de realizar algunos cambios en el Ciclo de Mejora con el objetivo de afianzar los conceptos relacionados con las cuestiones 1, 2 y 5 . El concepto de tensión superficial del agua (cuestiones 1 y 2) se podría afianzar ampliando la actividad de contraste de la práctica 1 en la primera sesión. Así, junto con el texto de Benjamin Franklin se podría entregar otro documento en forma de artículo científico o de artículo de divulgación en el que se hablase de la tensión superficial. Las cuestiones 4 y 5 no están directamente enfocadas en la práctica con la que están relacionadas (práctica 2), sino en el concepto que se pretende enseñar. Se podrían modificar las cuestiones para que los estudiantes asociasen más fácilmente el concepto trabajado, en este caso la tenacidad, con la práctica. Además, debido a que durante la realización de las prácticas se comprobó que los estudiantes conocían bien la mecánica del péndulo Charpy (tal 
como se ha indicado en el relato de las sesiones) se podría modificar la actividad de contraste de forma que el documento que se entregase estuviese relacionado con la tenacidad de distintos tipos de materiales.

\section{Evaluación del CIMA}

La experiencia con este ciclo de mejora ha sido muy buena, ya que los estudiantes estaban muy motivados. La idea de la no utilización del teléfono móvil durante las prácticas surgió a para evitar distracciones en unas prácticas en las que debían estar muy concentrados en la preparación de los diseños. Además, de esta forma se evitaba que buscasen posibles soluciones en internet, ya que uno de los principales objetivos del diseño metodológico es impulsar la creatividad.

\section{Cuestiones a mantener y cambios a introducir para un futuro Ciclo de Mejora más amplio}

En cuanto a cuestiones a mantener, considero que ha sido estimulante para los estudiantes tener que llevar a cabo ellos mismos el diseño de las prácticas a partir de los conceptos teóricos que debían trabajar y del procedimiento experimental que tenían que realizar. Por ello, la sensación ha sido que los estudiantes se han sentido más responsables de los resultados obtenidos que si hubiesen seguido una receta previamente elaborada por el profesor. La entrega de material de apoyo, como la carta de Benjamin Franklin, ha resultado ser una actividad de contraste muy interesante, ya que ha complementado las preguntas formuladas para los problemas planteados, y ha servido de orientación para llegar a la solución o soluciones posibles. He encontrado muy enriquecedores los debates con los estudiantes mientras diseñaban los experimentos porque ha permitido conocer muy bien sus ideas iniciales y 
cómo iban evolucionando hasta llegar al diseño correcto. Las puestas en común también han sido muy interesantes para dar visiones globales de las prácticas planteadas, se han sacado conclusiones de los resultados obtenidos y han servido para dar las respuestas a los dos problemas planteados.

En cuanto a los cambios a introducir, tal como se ha indicado en el epígrafe anterior, se podría trabajar con más profundidad el concepto de tensión superficial ampliando la actividad de contraste asociada a la práctica 1. De igual forma, también se podría modificar la actividad de contraste de la práctica 2 entregando un documento relacionado con la tenacidad de distintos tipos de materiales. También se podrían modificar las cuestiones 4 y 5 para que estuviesen más relacionadas con la parte experimental de la práctica 2. De esta forma la evaluación estará más enfocada aún en las prácticas realizadas. En cuanto a la realización de los experimentos, sería conveniente dar unas pautas más concretas en algunas etapas, para evitar, por ejemplo, que los estudiantes calienten a alta temperatura al realizar las disoluciones o cometan otros errores que afecten al resultado final. Aunque se ha preguntado al final de las prácticas a los estudiantes por su opinión acerca de éstas, sería interesante pasarles un cuestionario en el que plasmen sus opiniones, para que se tengan en cuenta en los siguientes grupos de prácticas.

\section{Aspectos de la experiencia que se pretenden incorporar a toda la práctica docente habitual}

- Conocer los modelos mentales de partida de los estudiantes antes de la realización del proceso de enseñanza-aprendizaje, mediante el empleo de cuestionarios y la realización de escaleras de aprendizaje que permitan considerar los posibles obstáculos de aprendizaje. 
- Explicar conceptos en forma de problemas que los estudiantes tengan que resolver a través de preguntas planteadas adecuadamente por el profesor.

- Elaborar mapas conceptuales y la secuencia de actividades que se va a aplicar.

- Hacer un diario de las sesiones para analizar la docencia impartida y evaluar si el modelo metodológico que se ha aplicado es correcto o si admite algún tipo de mejora.

- Utilización de cuestionarios finales para evaluar tanto el progreso de los estudiantes como el modelo metodológico, el profesor, la secuencia de actividades programada y el mapa de contenidos.

\section{Principios Didácticos argumentados que han guiado la experiencia y que deben permanecer en el futuro.}

El modelo metodológico que he adoptado en el Ciclo de Mejora que he experimentado está basado en los principios didácticos propuestos por D. Finkel. Se ha intentado provocar en los estudiantes el interés en resolver diferentes problemas, siguiendo la siguiente secuencia general de actividades (Finkel, 2008):

Planteamiento de un problema y de una o varias preguntas clave que guíen a los estudiantes hacia la resolución del problema. De cara a los estudiantes se plantea un problema, y de cara al profesor se plantean una serie de contenidos.

Realización de una actividad de contraste que sirva como apoyo para la resolución del problema. Se busca que los estudiantes reflexionen para que se cuestionen argumentos que tienen previamente asumidos. Así, la actividad de contraste provoca un conflicto con las ideas previas. 
Supervisar los debates de los estudiantes, que se organizan en grupos de trabajo, fomentando que piensen por ellos mismos. Antes de los debates, se debe dar un tiempo de reflexión a los estudiantes para que tengan en cuenta sólo sus ideas propias. Es importante considerar que los debates deben llevar finalmente a conclusiones que queden escritas en algún sitio (pizarra, diario de sesiones, etc.).

Realización de puestas en común en las que se proporcione la solución o soluciones al problema planteado. En las conclusiones no siempre se deben forzar consensos, sino reforzar las discrepancias para recoger diferentes puntos de vista.

Jornadas de Formación e Innovación Docente del Profesorado | № 2 (2019) Esta obra se distribuye con la licencia Creative Commons 


\section{Referencias bibliográficas}

Bain, K. (2004). Lo que hacen los mejores profesores universitarios. Valencia: Publicaciones de la Universidad de Valencia.

Franklin, B., Brownrigg, W. y Farish (1774). XLIV. Of the stilling of waves by means of oil. Extracted from sundry letters between Benjamin Franklin, LL. D. F. R. S. William Brownrigg, M. D. F. R. S. and the Reverend Mr. Farish. Philosophical Transactions of the Royal Society, 64, 445-460.

Bayraktar, E., Kaplan, D., Schmidt, F., Paqueton, H. y Grumbach, M. (2008). State of art of impact tensile test (ITT): Its historical development as a simulated crash test of industrial materials and presentation of new "ductile/ brittle" transition diagrams. Journal of Materials Processing Technology, 204, 313-326.

Porlán, R. (2017). Ensenanza Universitaria, Cómo mejorarla. Madrid: Morata.

Finkel, D. (2008). Dar clase con la boca cerrada, Valencia: Publicaciones de la Universidad de Valencia. 